\title{
Pathogenetic Substantiation of the Endothelial Dysfunction Correction in Ischemic Heart Disease of II Functional Class
}

\author{
Dzugkoev S.G. ${ }^{1,{ }^{*}}$ Dzugkoeva F.S. ${ }^{1}$ Garmash O.Yu. ${ }^{2}$ \\ ${ }^{1}$ Department of Pathobiochemistry, IBMI VSC RAS, Vladikavkaz, Russia \\ ${ }^{2}$ Functional diagnostics department of FSBI "SK MMTS” MZ, Beslan, Russia \\ *Corresponding author. Email: patbiochem@mail.ru
}

\begin{abstract}
The study focuses on redox processes in the development of vascular complications in coronary heart disease. The data showed the formation of active oxygen radicals, inducing the process of lipid peroxidation. The development of oxidative stress occurred with a decrease in the activity of the antioxidant protection enzymes of the cells. Inhibition of superoxidecismutase, catalase, and ceruloplasmin occurred. Under these conditions, there was a decrease in the content of total metabolites of nitric oxide, the main vasodilating factor. In violation of the bioavailability of the substrate L-arginine for the NO-forming enzyme, a violation of cholesterol metabolism played a role: an increase in total cholesterol, LDL, and a decrease of HDL cholesterol. In ischemic patients, these metabolic changes were accompanied by hypertension, impaired cardiac activity, which was manifested by ECG changes and anginal attacks. The obtained fundamental data served as the basis for the development and application of therapy, including atorvastatin and Q10, an inhibitor of cholesterol synthesis. Combined therapy with an HMG-CoA reductase inhibitor and coenzyme Q10 had an antioxidant effect and reduced the content of total cholesterol, LDL cholesterol, and such HDL cholesterol increase. These changes served as the basis for increasing the content of nitric oxide, vasodilation, and improving the clinical condition.
\end{abstract}

\section{Keywords: lipid peroxidation, antioxidant system, coronary heart disease, cholesterol}

\section{INTRODUCTION}

Over the past decade, researchers have been particularly interested in studying the mechanisms of endothelial dysfunction and its role in the development of vascular complications in many pathologies, including coronary heart disease. Metabolic disorders that occur during myocardial ischemia are a leading link in the pathogenesis of coronary heart disease, its clinical manifestations. Developing lipid peroxidation is a pathogenetic link in endothelial dysfunction and a decrease in the concentration of NOvasodilating factors. Current data presented in the literature indicate the critical role of endothelial dysfunction in the development of vascular complications [1-3].

The article aimed to study the role of redox processes, NO-producing endothelial function, and cholesterol metabolism in the development of endothelial dysfunction in patients with coronary heart disease. To achieve this goal, 75 patients with coronary heart disease of the II functional class and 30 healthy individuals were examined, who made up the control group on the basis of the Federal State Budgetary Institution "North Caucasian Multidisciplinary Medical Center of the Russian Federation" and the Department of Pathobiochemistry of IBMI in accordance with the contract. The average age in patients was $50.1 \pm 10$ years, the duration of the disease from 1-5 years or more. The analysis included the clinical status and results of biochemical methods carried out in the department of pathobiochemistry.

\section{METHODS AND MATERIALS}

The studies were conducted in a clinic and based on the Department of Pathobiochemistry, IBMI VSC RAS. Clinical material was analyzed in patients with coronary heart disease of functional class II. The intensity of lipid peroxidation was determined by the concentration of malondialdehyde in the erythrocyte hemolysate [4]. The state of the antioxidant system: superoxide dismutase [5], catalase [6], the concentration of ceruloplasmin [7]. The concentration of total metabolites of nitric oxide (NOx) express method in the modification of V.A. Metelskaya [8]. Violation of cholesterol metabolism was judged by total cholesterol, LDL, cholesterol in high-density lipoproteins, TAG. An ECG study was performed, daily monitoring with the help of an ECG logger using MicrovitMT-101 halter, blood pressure to verify the diagnosis of coronary heart disease.

The following were evaluated: ECG review, ST trend, mean blood pressure, hypertension time index, the daily 
rhythm of blood pressure, morning rise in blood pressure, variability. Used bicycle ergometry-ECG using dosed physical activity. Using Microsoft Excel 2006, statistical processing of the results was carried out, which were presented as mean values (Mean) and meant errors (SEM). The Student's t-test helps to investigate the statistical significance of differences between groups of patients was checked, $\mathrm{p}<0.05$ was considered the level of statistical significance. The correlation analysis is carried out.

\section{RESULTS}

In patients with coronary heart disease, there is an intensification of oxidative processes, an increase in the concentration of malondialdehyde in red blood cells. Analysis of the antioxidant system activity results showed a decrease in catalase superoxide dismutase activity and ceruloplasmin concentration in patients with coronary heart disease. Oxidative stress develops, which is accompanied by a decrease in the concentration of total NO metabolites. An important mechanism limiting the damaging effect of CPP products is the antioxidant system, which is found to be insolvent. Therefore, oxidative stress assumes a systemic character. Our studies have found that developing oxidative stress leads to disruption of the NOproducing function of the endothelium and a decrease in the content of nitric oxide (NO) $[9,10]$. It is formed from $\mathrm{L}$-arginine with the participation of the enzyme synthase nitric oxide (NO synthase), with the formation of an oxygen molecule and L-citrulline [11-13]. A manifestation of endothelial dysfunction is a violation of blood pressure - hypertension - a risk factor for myocardial ischemia. Another critical factor in the development of ischemic heart disease was impaired cholesterol metabolism, hypercholesterolemia, as well as the oxidative

Table 1 The dynamics of blood lipid profile in patients with coronary heart disease of the II functional class against the background of complex therapy.

\begin{tabular}{|c|c|c|c|c|c|c|}
\hline \multirow[t]{2}{*}{ Study group } & \multirow{2}{*}{ 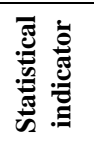 } & \multicolumn{5}{|c|}{ INDICATORS OF LIPID SPECTRA OF BLOOD } \\
\hline & & $\begin{array}{c}\text { General } \\
\text { cholesterol }\end{array}$ & $\begin{array}{c}\text { Common } \\
\text { Triglycerides }\end{array}$ & HDL cholesterol & LDL cholesterol & $\begin{array}{c}\text { Atherogenic } \\
\text { coefficient }\end{array}$ \\
\hline \multirow[t]{3}{*}{ Group $1(n=30)$} & M & 4.30 & 1.23 & 1.46 & 2.68 & 3.3 \\
\hline & $\mathrm{m}$ & 0.11 & 0.05 & 0.02 & 0.17 & 0.11 \\
\hline & $\mathrm{p}<$ & 0.001 & 0.001 & 0.001 & 0.001 & 0.001 \\
\hline \multirow[t]{3}{*}{ Group $2(n=25)$} & $\mathrm{M}$ & 6.54 & 3.05 & 0.75 & 5.50 & 5.54 \\
\hline & $\mathrm{m}$ & 0.10 & 0.11 & 0.01 & 0.12 & 0.09 \\
\hline & $\mathrm{p}<$ & 0.001 & 0.001 & 0.001 & 0.001 & 0.001 \\
\hline \multirow[t]{3}{*}{ Group $3(n=25)$} & $\mathrm{M}$ & 5.02 & 2.1 & 1.14 & 3.32 & 2.96 \\
\hline & $\mathrm{m}$ & 0.05 & 0.02 & 0.02 & 0.06 & 0.05 \\
\hline & $\mathrm{p}<$ & 0.001 & 0.001 & 0.001 & 0.001 & 0.001 \\
\hline \multirow[t]{3}{*}{ Group $4(n=25)$} & M & 4.87 & 1.94 & 1.21 & 2.97 & 3.87 \\
\hline & $\mathrm{m}$ & 0.05 & 0.02 & 0.01 & 0.02 & 0.53 \\
\hline & $\mathrm{p}<$ & 0.001 & 0.001 & 0.001 & 0.001 & 0.001 \\
\hline
\end{tabular}

Note:

$1111)-\mathrm{p}<0.001 ; 111)-\mathrm{p}<0.001 ; 11)-\mathrm{p}<0.02 ; 1)-\mathrm{p}<0.05$, the reliability of the 1 st group relative to the control.

$2222)-\mathrm{p}<0.001 ; 222)-\mathrm{p}<0.01 ; 22)-\mathrm{p}<0.002 ; 2)-\mathrm{p}<0.05$, the reliability of the 1 st group relative to the 2nd group.

$3333)-\mathrm{p}<0.001 ; 333)-\mathrm{p}<0.01 ; 33)-\mathrm{p}<0.02 ; 3)-\mathrm{p}<0.05$, the reliability of the 2 nd group relative to the 3rd group. modification of atherogenic lipoproteins during their influence of lipid peroxidation products [14-16]. Besides, inadequate utilization by tissues of cells supports its increased content in the blood. An increase in the content of lipid peroxidation products leads to inhibition of rostacyclin synthesis and the induction of smooth muscle cell proliferation. Collagen synthesis occurs; the lipid stain is covered with connective tissue and atherosclerotic plaque forms. The described disorders of cholesterol metabolism in atherosclerosis occur against the background of the development of a deficiency syndrome vital antioxidant enzymes $[17,18]$. When studying the lipid spectrum of blood in patients with coronary heart disease of the II functional class, an increase in the content total cholesterol and cholesterol of lipoproteins was revealed, while lowering HDL cholesterol (Table 1). Impaired cholesterol metabolism causes atherogenic changes in the endothelium of the vessel. Perhaps changes in this plan are involved in reducing the bioavailability of L-arginine for the NO-forming enzyme.

Therefore, our data showing violations in cholesterol metabolism indicate hypercholesterolemia and hyper- $\beta$ lipoproteinemia. We draw attention to the possibility of peroxidation of lipoproteins under conditions of oxidative stress. Based on this possibility, it can be assumed that the atherogenicity of lipoproteins compared with native ones is increasing. In standard therapy, drugs did not cause significant inhibition of the intensity of lipid peroxidation according to malondialdehyde in patients with coronary heart disease of functional class II with angina pectoris. Some positive dynamics of the clinical picture was noted, but metabolic indices did not come close to the data of control individuals. formation and disturbance in the bloodstream under the 
The positive dynamics of the clinical condition of patients with coronary heart disease with functional class II angina was manifested after 3 months of traditional treatment. The activity of the antioxidant protection enzymes of the cells (superoxide dismutase and catalase), as well as the concentration of Ceruloplasmin, did not change enough during therapy. Therefore, the data characterizing the state of the lipid peroxidation system, the antioxidant protection of cells in this group of patients with coronary heart disease, remained above the norm. Our results are presented in Table 2.

Thus, the results obtained were the basis for the development and use of a new methodological approach for the correction of endothelial dysfunction by the cholesterol metabolism regulator. The use of an inhibitor of the critical reaction for the synthesis of cholesterol HMG-CoA reductase atorvastatin in combination with standard drugs helped to reduce total cholesterol, lipoprotein cholesterol, and increase HDL cholesterol. At the same time, it had a stimulating effect on the activity of the antioxidant system: superoxide dismutase, catalase, Ceruloplasmin. Consequently, combination therapy with hemodynamic drugs and an atoris inhibitor of HMG-CoA reductase contributed to a significant decrease in the total cholesterol of atherogenic fractions and an increase in antiatherogenic fractions.

These changes occur with inhibition of lipid peroxidation. Inhibition of lipid peroxidation as a result of therapy has become possible due to a specific positive effect on the antioxidant defense system of cells. In particular, this effect on its enzymatic component can be observed, which is expressed in a significant increase in the activity of the antioxidant protection enzymes of cells: superoxide dismutase by $34.27 \%$, catalase by $11.16 \%$, Ceruloplasmin by $12.99 \%$ ( $\mathrm{p}<0.001$ ). However, it should be noted that the enzyme activity indicators level of the antioxidant system in specific values was lower

The use of atoris in combination with Coenzyme Q10 against the background of standard therapy led to a more pronounced decrease in the concentration of malondialdehyde compared with indicators and against the background of the complex with the inhibitor of HMGCoA reductase atoris. The system of antioxidant cell protection has also undergone significant changes since the absolute values of the activity of superoxide dismutase, catalase, the concentration of Ceruloplasmin, NO have become significantly higher. It can be noted that these values approached the control values. Coenzyme Q10 showed its pronounced antioxidant properties in patients with coronary artery disease. Inhibition of lipid peroxidation due to the activation of antioxidant system enzymes contributed to an increase in nitric oxide content to control values.

Thus, the reduced activity of enzymes of the antioxidant system almost wholly recovered to control parameters in patients with complex treatment with atoris and coenzyme Q10. Under conditions of balance in the system of "lipid peroxidation - antioxidant system," the content of nitric oxide, the primary vasodilator, increased. Based on the data obtained, it should be noted that coenzyme Q10 is a critical electron carrier in the respiratory chain, takes electrons from different sources, and provides adequate biological oxidation, as well as the conjugation of oxidation with oxidative phosphorylation. The conjugation of these processes generates an electrochemical gradient on the inner mitochondrial membrane, which is a factor in restoring the energy supply and the functional ability of the myocardium. Biologically active vitamin $\mathrm{E}$, which is essential for lipoproteins, is maintained in a reduced state, also with the participation of coenzyme Q10.

The effect of complex therapy with atoris and coenzyme Q10 on the activity of antioxidant enzymes in red blood cells and blood plasma in patients with coronary heart disease is analyzed. In general, this analysis shows not only the approximation of parameters to the level of indicators of healthy volunteers but even exceeds them compared to the action of one atoris. These results support the effectiveness of the healing effect of combination therapy, including the antioxidant system in patients with coronary heart disease. The effect of action developed throughout therapy. It should be assumed that the inhibition of lipid peroxidation and the reduction of atherogenic lipoproteins helps restore the complementarity of apo-B-100 LDL to its receptors, which improves the affinity of their interaction (Table 2).

Table 2 Indicators of pro-and antioxidant systems in patients with coronary heart disease of functional class II during treatment.

\begin{tabular}{|c|c|c|c|c|c|}
\hline \multirow[t]{2}{*}{ Study group } & \multicolumn{5}{|c|}{ Indicators of lipid peroxidation and antioxidant system before and after 3 months of treatment } \\
\hline & $\begin{array}{c}\text { malondialdehyde, } \\
\mathrm{nmol} / \mathrm{ml}\end{array}$ & $\begin{array}{c}\text { superoxide } \\
\text { dismutase, } u / \mathbf{m g}\end{array}$ & NO, $\mu \mathrm{mol}$ & $\begin{array}{c}\text { Ceruloplasmin } \\
\text { min, mg/l }\end{array}$ & $\begin{array}{c}\text { Catalase, } \\
\mathrm{nmol} / \mathrm{g} / \mathrm{Nv}\end{array}$ \\
\hline Control group $(n=30)$ & $17.3 \pm 0.23$ & $67.4 \pm 0.52$ & $68.4 \pm 0.59$ & $262.5 \pm 3.99$ & $228.8 \pm 2.33$ \\
\hline Group $1(n=25)$ & $\left.31.7 \pm 0.15^{1111}\right)$ & $\left.35.6 \pm 0.79^{1111}\right)$ & $\left.38.90 .73 \pm^{1111}\right)$ & $\left.149.3 \pm 3.12^{1111}\right)$ & $\left.160.4 \pm 1.53^{1111}\right)$ \\
\hline Group $2(n=25)$ & $\left.25.6 \pm 0.09^{2222}\right)$ & $\left.47.8 \pm 0.54^{2222}\right)$ & $\left.54.2 \pm 0.81^{2222}\right)$ & $\left.194.0 \pm 0.89^{2222}\right)$ & $\left.183.1 \pm 0.66^{2222}\right)$ \\
\hline Group $3(n=25)$ & $\left.20.10 \pm 0.08^{3333}\right)$ & $\left.61.2 \pm 0.93^{3333}\right)$ & $\left.60.2 \pm 0.70^{3333}\right)$ & $\left.239.7 \pm 1.04^{3333}\right)$ & $\left.201.7 \pm 0.90^{3333}\right)$ \\
\hline
\end{tabular}

Note:

Group 1 - Control - healthy individuals.

Group 2 - patients with coronary heart disease at admission.

Group 3 - patients with coronary heart disease who received standard therapy + atorvastatin.

Group 4 - patients with coronary heart disease who received complex therapy with coenzyme Q10.

$\left.\left.\left.\left.{ }^{1111}\right)-\mathrm{p}<0.001 ; 111\right)-\mathrm{p}<0.001 ; 11\right)-\mathrm{p}<0.02 ; 1\right)-\mathrm{p}<0.05$, the reliability of the 1 st group relative to the control.

$\left.\left.\left.\left.{ }^{2222}\right)-\mathrm{p}<0.001 ; 222\right)-\mathrm{p}<0.01 ; 22\right)-\mathrm{p}<0.002 ; 2\right)-\mathrm{p}<0.05$, the reliability of the 1 st group relative to the 2 nd group.

$\left.\left.\left.\left.{ }_{3333}\right)-\mathrm{p}<0.001 ; 333\right)-\mathrm{p}<0.01 ; 33\right)-\mathrm{p}<0.02 ; 3\right)-\mathrm{p}<0.05$, the reliability of the 2 nd group relative to the 3rd group. 
As can be seen from Table 2, statistically significantly changed indicators of blood cholesterol metabolism during treatment ( $\mathrm{p}<0.001, \mathrm{p}<0.01)$, and approached the control figures after a month of taking complex therapy. Moreover, indicators such as total cholesterol decreased by $16 \%$, HDL cholesterol reached the control figures, which indicates a pronounced antiatherogenic effect of this combination.

\section{CONCLUSION}

The obtained results demonstrate the development of oxidative stress on the background of combined treatment in patients with coronary heart disease of the II functional class. There was a significant increase in lipid peroxidation products and inhibition of the activity of enzymes of the antioxidant system: superoxide dismutase for intoxication syndrome and superoxide dismutase, catalase, and ceruloplasmin concentration in coronary heart disease. Changes in redox processes have led to a decrease in the concentration of total metabolites of nitric oxide, the pathogenetic link of endothelial dysfunction. Cholesterol metabolism: hypercholesterolemia and hyper- $\beta$ lipoproteinemia contributed to endothelial dysfunction. LDL becomes even more atherogenic under conditions of oxidative stress. The affinity of lipoprotein particles to their receptors is disrupted, and phagocytes absorb them. There is an accumulation of lipids in the endothelium, which leads to atherogenesis. Changes in the structure of the endothelium disrupt the bioavailability of L-arginine for eNOS and cause a decrease in the content of nitric oxide. The use of an inhibitor of the synthesis of cholesterol- $\beta$-HMG-CoA reductase (atorvastatin and coenzyme Q10) has an antioxidant effect in combination with standard therapy and reduces the content of total cholesterol and its atherogenic fraction. Such a plan, metabolic changes contribute to the restoration of endothelial function and increase nitric oxide, the primary vasodilator, which indicates the effectiveness of the treatment of patients with coronary heart disease II functional class.

\section{REFERENCES}

[1] O.V. Kostenko, Features of vascular lesions and endothelial disorders in various forms of coronary heart disease, Bull. of new med. technol. XIV(2) (2007) 41-43.

[2] R.G. Oganov, Diagnosis and correction of lipid metabolism disorders for the prevention and treatment of atherosclerosis (Russian recommendations IV revision), Cardiovascular Therapy and prevent. 8(6(3)) (2009) 58.

[3] T. Shoji, J. Nishizawa, M. Fukumoto, K. Shimamura, Inverse relationship between circulating oxidized low-density lipoprotein and antioxLDL antibody levels in healthy subjects, Atherosklerosis 14 (2000) 171-177.

[4] T. Asakawa, S. Matsushita, Coloring conditions of thiobarbituric acid test, for detecting lipid hidroperoxides, Lipids 15(5) (1980) 137-140.

[5] T.V. Sirota, A new approach to the study of the autooxidation of adrenaline and its use to measure superoxide dismutase activity, Quest. of med. chem. 3 (1999) 263-272.

[6] M.A. Korolyuk, L.I. Ivanova, I.G. Mayorova, Method for the determination of catalase activity, Laboratory work 1 (1988) 16-19.

[7] V.S. Kamyshnikov, Determination of the content of ceruloplasmin activity, Handbook of clinical and biochemical studies and laboratory diagnostics, vol. 2, 2003, pp. 71-79.

[8] A.V. Metelskaya, N.G. Humanova, Screening method for determining the level of nitric oxide metabolites in human serum, Laboratory med. 7 (2005) 19-24

[9] M.V. Cossack, Endothelial dysfunction in the pathogenesis of ischemic stroke in patients with arterial hypertension, Acta Biomed. Sci. 2(60) (2008) $17-18$.

[10] V.S. Zadiochenko, T.V. Adasheva, A.P. Sandomierz, Endothelial dysfunction, and arterial hypertension: therapeutic options, Breast cancer 10(1) (2002) 15-21.

[11] P.V. Bovtyushko, S.L. Grishaev, E.A. Filippov et al., Markers of subclinical atherosclerosis in assessing the ten-year risk of developing cardiovascular events, Cardio Somatica 4 (2015) 12-16.

[12] S.Yu. Matusevich, K.A., Karuzin, Oxidative stress and its role in the formation of maladaptation and pathology, Bioradicals and antioxidants 2(2) (2015) $15-18$.

[13] V.Z. Lankin, A.K. Tikhadze, Yu.N. Belenkov, Free radical processes are normal and in pathological conditions, RKNPK, Moscow, 2001, 78 p.

[14] B.M. Lipovetsky, Dyslipidemia, atherosclerosis, and their relationship with coronary heart and brain disease, St. Petersburg, 2012, 130 p.

[15] I.Yu. Lukyanova, Optimization, treatment, and prevention of the progression of chronic heart failure based on the use of drugs with antioxidant and anxiolytic effects, Psychopharmacol. and biolog. Narcol. 7 (2007) 1-17.

[16] Yu.R. Tikhomirova, K.N. Kontorshchikova, Indicators of lipid metabolism and oxidative modification of proteins in metabolic disorders, Bioradicals, and antioxidants 2(1(2)) (2015) 62-66.

[17] A.N. Zakirova, N.E. Zakirova, The role of lipid peroxidation, antioxidant protection, and rheological disorders in the development of coronary heart disease, Russ. J. of Cardiol. 2 (2006) 24-27. 
[18] V.Z. Lankin, A.K. Tikhase, V.I. Fireplace et al., In vivo intensification of free radical oxidation of lowdensity lipoproteins in the blood plasma of patients with coronary heart disease during treatment with
GMK-CoA reductase inhibitor pravastatin and suppression of lipoperoxidation by ubiquinone Q10, Bull. exper. biol. and honey. 129(2) (2000) 176-179. 